\title{
Expression and Analysis of Microtus fortis Against Schistosoma japonicum CD72
}

\author{
Yang Xiang ${ }^{1}$ and Junjian $\mathrm{Hu}^{2}$ \\ 1. College of Chemistry and Chemical Engineering, Hunan Institute of Science and Technology, Yueyang 414006, China \\ 2. Hunan Provincial Institute of Schistosomiasis Control, Yueyang 414006, China
}

Received: September 30, 2014 / Accepted: September 30, 2014 / Published: September 30, 2014.

\begin{abstract}
The total RNA was extracted from Microtus fortis liver tissue which before being infected and after being infected $10 \mathrm{~d}$ and $15 \mathrm{~d}$ by the Schistosoma japonicum cercariae. Using rattus norvegicus CD72 gene probes were used to hybridize analysis of CD72 difference expression in the Microtus fortis liver tissues which were infected with Schistosoma japonicum before and after being infected. At the same time, the cDNA sequence and encoded amino acid sequence of the rattus norvegicus CD72 gene and CD72 protein structural domains were analyzed by using bioinformatics. The results showed that the CD72 expression levels in the liver tissue of Microtus fortis after being infected was significantly higher than before being infected. The rattus norvegicus CD72 cDNA sequence of a total length is $1479 \mathrm{bp}$ and encode 364 amino acid residues and rattus norvegicus CD72 protein containing a CD72 superfamily domain.
\end{abstract}

Key words: Microtus fortis, Schistosoma japonicum, CD72, expression, analysis.

\section{Introduction}

Schistosomiasis is the world's second-largest parasitic disease after malaria, serious harm to human health. At least 76 countries and regions to be endangered, some 600 million people are threatened, 200 million people are infected, and China is one of the hardest hit of schistosomiasis. The Chinese pop schistosomiasis mainly is schistosomiasis japonica, a population of 11.612 million people infected schistosomiasis in China, a population of 130 million people threatened by schistosomiasis in the world. Schistosomiasis in China are mainly distributed in Jiangsu, Hunan, Hubei, Sichuan and Yunnan Province, Dongting Lake area as a national one of heavy disaster area, infected population occupies countrywide 1/4. Microtus fortis is a no suitable host for Schistosoma japonicum, with natural completely against schistosomiasis characteristics [1]. Oriental vole

Corresponding author: Yang Xiang, Ph.D., professor, research field: molecular and cell biology. E-mail: xiangyangbio@126.com. distribution in China is mainly concentrated in the Yangtze River Basin, especially in endemic area of schistosomiasis japonica in Dongting Lake Island, is a dominant rat in the area. Schistosoma japonicum growth stagnation after infected Microtus fortis $12 \mathrm{~d}$, and all Schistosoma japonicum demise after infected Microtus fortis 20 to $28 \mathrm{~d}$ in vivo [2]. The major organ Schistosoma japonicum was perished is in the liver [3]. Experiments showed that rat, rattus, the bandicoot rat, Rattus norvegicus, Hainan, roof rats to infection with Schistosoma japonicum susceptibility from high to low [4]. This reveals the different rat species of Schistosoma japonicum have different resistance, rat as unsuitable host of Schistosoma japonicum. So far, almost all of the schistosome infection animal models are positive model, but Microtus fortis to infection with Schistosoma japonicum model is a negative model. Therefore, use of Microtus fortis in natural resistance to Schistosoma japonicum infection in animal models, Chinese scientists did a lot of research work on Microtus fortis in humoral immunity and 
cellular immunity [5], natural antibody associated antigen gene [6], screening resistance gene [7], gene expression profiling using microarrays of Microtus fortis to infection with Schistosoma japonicum and differences in gene [8] and function research. CD72 is a transmembrane protein is macrophage migration inhibitory factor (MIF) receptor molecules, and related to a variety of human diseases and parasitic infections [9]. This paper compares the Fortis to infection with Schistosoma japonicum, expression difference and bioinformatics analysis of CD72, for further research of CD72 in Microtus fortis against schistosomiasis mechanism lays a foundation.

\section{Materials and Methods}

\subsection{Animal}

The Microtus fortis, as indoor propagation of the Dongting Lake in Hunan, was provided by the Hunan Provincial Institute of Schistosomiasis Prevention and Control Research. Schistosoma japonicum cercariae, provided by the Hunan Provincial Institute of Schistosomiasis Prevention and Control Research, were used artificial quantitative infection experiment.

\subsection{Sample Collection and RNA Extraction}

1100 Schistosoma japonicum were used infected fortis, and total RNA was extracted from Microtus fortis liver tissue and positive control after infection $10 \mathrm{~d}$ and $15 \mathrm{~d}$.

\subsection{Northern Blot Experiments}

The experimental chip hybridization experiment operated as follows: Rat (Rattus norvegicus) CD72 gene probes were used hybridization with Microtus fortis uninfected and infected after $10 \mathrm{~d}$ and $15 \mathrm{~d}$ the liver tissue samples mRNA, respectively.

\subsection{Bioinformatics Analysis}

U.S. National Center for Biotechnology Information NCBI EST database (http://www.ncbi.nlm. Nih.gov/) and

Unigene

database
(http://www.ncbi.nlm.nih.gov/HomoloGene/nih.gov/U nigene) were used for bioinformatics analysis. Identification of the Reading frame: Using the ExPASy server Translate and NCBI ORF finder. The protein domain analysis: protein blast in NCBI (http://blast.ncbi.nlm.nih.gov/Blast.cgi).

\section{Results}

3.1 The RNA Extraction from the Microtus fortis Liver Tissue after Infected and not Infected Schistosoma Japonicum

The total RNA were extracted according to the description extraction in Molecular Cloning from Microtus fortis liver tissue infected with Schistosoma japonicum ago and after infected $10 \mathrm{~d}$ and $15 \mathrm{~d}$. Sample mRNA in each lane approximately $2 \mu \mathrm{g}$ (Fig. 1).

(1) The Microtus fortis not infected with Schistosoma japonicum;

(2) The Microtus fortis liver tissue after $10 \mathrm{~d}$ infected with Schistosoma japonicum;

(3) The Microtus fortis liver tissue after $15 \mathrm{~d}$ infected with Schistosoma japonicum.

3.2 The Differential Expression Analysis of CD72 in Microtus fortis Liver Tissue Infected with Schistosoma japonicum after $10 d$ and $15 d$ with Normal Control Group

The expression of CD72 in Microtus fortis liver tissue infected with Schistosoma japonicum after $10 \mathrm{~d}$ and $15 \mathrm{~d}$ significantly increased (Fig. 2) than the normal control group, after Northern blot analysis with rat $\mathrm{CD} 72$ gene probe.

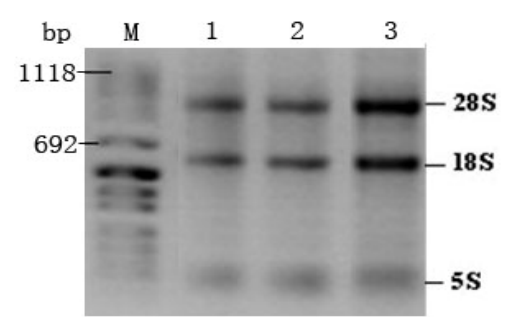

Fig. 1 Total RNA extraction results from Microtus fortis liver tissue after Infected and not Infected with Schistosoma japonicum. 


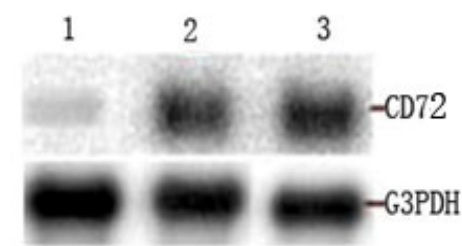

Fig. 2 Northern blot analysis of CD72.

(1) The Microtus fortis liver tissue not infected with Schistosoma japonicum;

(2) The Microtus fortis liver tissue infected with Schistosoma japonicum after $10 \mathrm{~d}$;

(3) The Microtus fortis liver tissue infected with Schistosoma japonicum after 15 d (B) P3GDH.

\subsection{Bioinformatics Analysis of the Complete Open} Reading Frame of CD72 Gene

The full length cDNA sequence of rat CD72 contains 1479 bp, encoding 364 amino acid residues (Fig. 3).

\subsection{Bioinformatics Analysis of CD72 Protein Domain}

Rat CD72 proteins contain 364 amino acid residues and contain a CD72 superfamily domains (Fig. 4).

\section{Discussion}

Schistosomiasis is the world's second-largest parasitic disease after malaria, serious harm to human health. China is one of the hardest hit of schistosomiasis. There are 1.1612 million people were threatened by Japanese schistosomiasis in China. Microtus fortis has natural anti-Schistosoma japonicum capacity is Schistosoma japonicum non-suitability host. Rat CD72 gene probe was used Northern blot analysis with the Microtus fortis liver tissue mRNA. The results showed the CD72 gene expression significantly up-regulated in the Microtus fortis liver tissue infected with Schistoso majaponicum after $10 \mathrm{~d}$ and $15 \mathrm{~d}$ compare with contral. The CD72 is Macrophage uptake of oxidized low-density lipoprotein (OX-LDL) receptor molecules, involved in immune regulation, which show that the immune system to get a positive response after Microtus fortis infected with Schistosoma japonicum. CD72 has a role against Schistosoma japonicum.

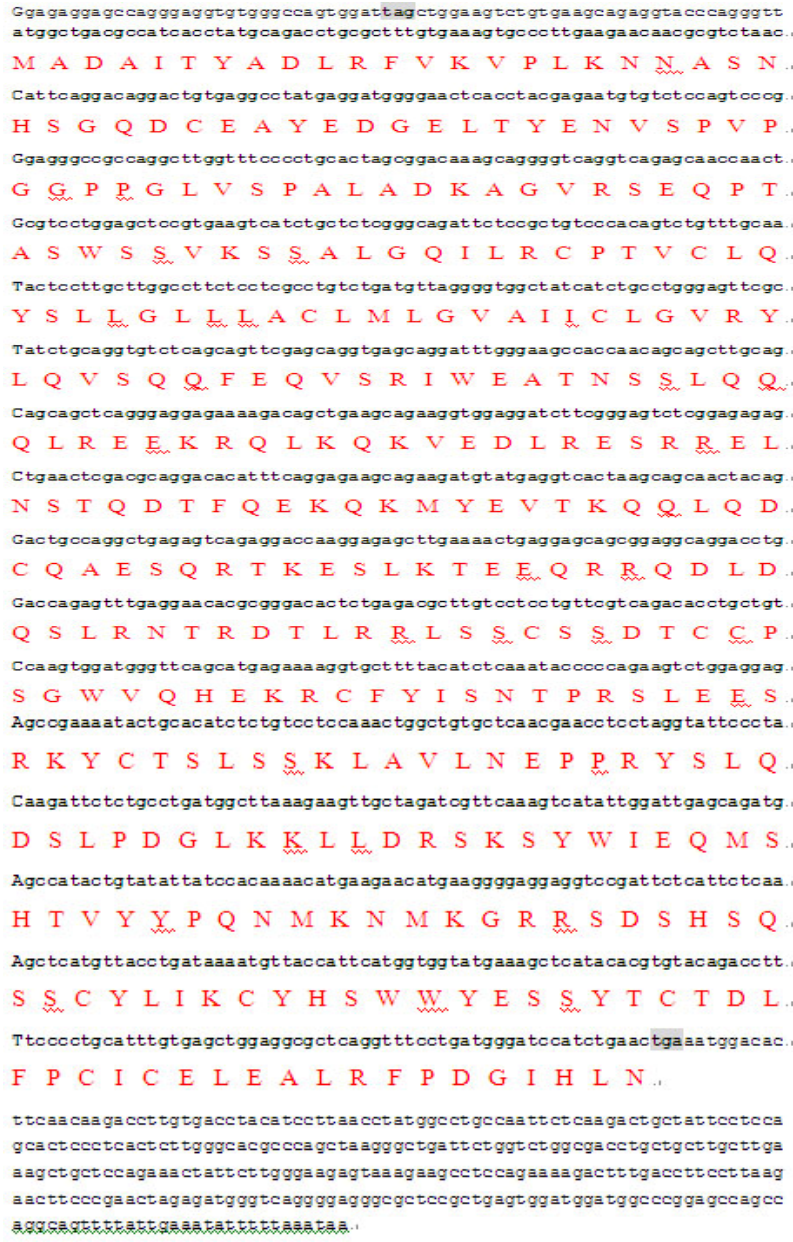
a agct get ccagaaactatt ct tggga agag taa agaagcctccagaa aagactt tgaccttcct ta ag

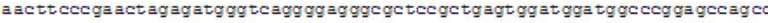

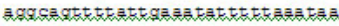

Fig. 3 The amino acid sequence encoded by the rat CD72 gene cDNA sequence.

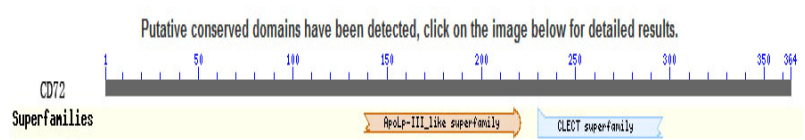

Fig. 4 Analysis of the rat CD72 protein domain.

\section{Conclusions}

In summary, The results of the study demonstrated that rat CD72 gene probe was used Northern blot analysis with the Microtus fortis liver tissue mRNA and the CD72 gene expression significantly up-regulated in the Microtus fortis liver tissue infected with Schistoso majaponicum after $10 \mathrm{~d}$ and $15 \mathrm{~d}$ compare with control.

\section{Acknowledgments}

This work was supported by grants from the 
Department of Education key project of Hunan Province, China (No: 09A035).

\section{References}

[1] He, Y., Luo, X., Zhang, X., Yu, X., Lin, J., and Li, Y. 1999. "Immunological Characteristics of Natural Resistance in Microtus fortis to Infection with Schistosoma japonicum." Chin Med J. 112 (7): 649-654.

[2] Yu, Y. J., Hu, W. X., Wang, Y., Su, Z. J., Yi, X. Y., and Peng, X. H. 2003. "The Primary Studies on Breeding Microtus fortis as Laboratory Animal and Protecting Their Germ Plasm." Laboratory Animal Science and Management 20: 13-17.

[3] Ll, H., He, Y. Y., Lin, B. F., Lin, J. J., Chen, Y. J., and Wang, Y. J. 2001. "The Preliminary Research of Microtus fortis Superinfection Schistosoma japonicum." Chinese Journal of Veterinary Parasitology 9 (3): 11-17.

[4] Liu, J. S., Yong, S. M., Chen, P. C., Liu, Z. H., and Mo, G. Y. 1992. "The Several Rodents Artificial Experimental Observation of Schistosoma japonicum Infection." Journal of Schistosomiasis Control in China 4
(6): 350-351.

[5] Jiang, S. H., Wei, M. X., Lin, J. J., Pan, C. E., Qiu, Q. W., and He, Y. Y. 2008. "Research of IgG3 Antibody Anti-schistosomiasis." Chinese Journal of Parasitology and Parasitic Diseases 26 (10): 34-36.

[6] Sun, Y., Sun, H., Jia, R. C., Liu, J. M., Yuan, C. X., and Shi, Y. J. 2008. "Relative Target Genes Screening of Microtus fortis Against Schistosoma japonicum." Chinese Journal of Schistosomiasis Control 20 (1): 26-31.

[7] Qin, Z. Q., Hu, W. X., Wu, G. J., Xu, B., Shen, Q. X., and Gong, Q. 2004. "Oriental Vole Bone Gene Pool and Schistosoma japonicum Resistance Related Gene Screening." Life Science Research 8 (4): 333-338.

[8] Sun, J., Lin, J. J., Cheng, G. F., Li, H., Shi, Y. J., and Lu, K. 2004. "Gene Difference Expression Research Profiling using Microarrays to Study Oriental Voles and Mice Infected before and after with Schistosoma japonicum." Journal of Peking University (natural science edition) 40 (4): 532-537.

[9] Kleemann, R., Hausser, A., and Geiger, G., et al. 2000. "Intracellular Action of the Cytokine MIF to Modulte AP1 Activity and the Cell Cycle through Jab1." Nature 408 (6809): 211-216. 